\title{
ESSENTIAL SELFADJOINTNESS OF CERTAIN PARTIAL DIFFERENTIAL OPERATORS ON $R^{n}$
}

\author{
A. DEVINATZ ${ }^{1}$
}

\begin{abstract}
Sufficient conditions are given on the coefficients of a second order, semibounded, formally selfadjoint differential operator on $R^{n}$, not necessarily elliptic, so that the closure of the operator restricted to $C_{0}^{\infty}\left(R^{n}\right)$ is selfadjoint. The results are based on A. E. Nussbaum's notion of quasianalytic vectors.
\end{abstract}

1. Introduction. In this note we shall study the essential selfadjointness of the minimal operator associated with a formally symmetric partial differential operator of the form

$$
L u=\sum_{|\alpha| \leqslant 2} a_{\alpha}(x) \partial^{\alpha} u .
$$

The coefficients shall be matrix valued and are defined on $R^{n}$. The exact smoothness and growth conditions on the coefficients will be delineated in the statement of the theorem.

Schrödinger type operators, i.e., those operators for which $L$ is elliptic and the $a_{\alpha}$ are scalars, have been the subject of intensive investigations by many mathematicians during the past two to three decades. On the other hand the case where $L$ is nonelliptic seems not to have received much attention. We shall here make a start in this direction.

The proof of our theorem is based upon a result of A. E. Nussbaum [3] concerning the essential selfadjointness of symmetric operators which contain in their domains total sets of quasi-analytic vectors. His theorem states: If $S$ is a semibounded symmetric operator on a Hilbert space $H$, and if there exists a total set of vectors $u \in \cap\left\{D\left(S^{n}\right): n \geqslant 1\right\}$ so that $\sum_{n=1}^{\infty}\left\|S^{n} u\right\|^{-1 / 2 n}=\infty$, then $S$ is essentially selfadjoint.

2. The main theorem. The coefficients $a_{\alpha}$ in (1.1) shall be $m \times m$ matrix valued functions defined on $R^{n}$. We shall designate by $L_{m}^{2}\left(R^{n}\right)$ the space of (equivalent classes of) $m$-dimensional vector valued functions on $R^{n}$ for which $\|u\|^{2}=\int_{R^{n}}|u|^{2} d x<\infty$. The inner product shall be taken as

$$
(u \mid v)=\int u \cdot v d x
$$

Received by the editors November 25, 1974.

AMS (MOS) subject classifications (1970). Primary 35P99; Secondary 35R99.

Key words and phrases. Partial differential operators, essential selfadjointness, quasi-analytic vectors.

${ }^{1}$ Research partially supported by NSF Grant GP-37491X1. 
where $u \cdot v=\sum_{1}^{m} u_{j} \bar{v}_{j}$, and of course $|u|^{2}=u \cdot u$.

We shall designate by $L_{0}$ the closure in $L_{m}^{2}\left(R^{n}\right)$ of the operator $L$ acting on $C_{0}^{\infty}\left(R^{n}\right)$, and call it the minimal operator associated with $L$. We are, of course, supposing that the $a_{\alpha}$ are locally square integrable so that this makes sense.

In what follows we shall use $|x|$ and $x \cdot y$ to designate the usual Euclidean norm and dot product for elements of $R^{n}$. If $a_{\alpha}$ is a matrix by $\left|a_{\alpha}\right|$ we mean any convenient norm for $a_{\alpha}$. We shall often designate $\partial^{\alpha} f$ by $f^{(\alpha)}$.

THEOREM. Suppose the coefficients of the formally selfadjoint operator L of (1.1) are in $C^{\infty}\left(R^{n}\right)$. The minimal operator $L_{0}$ associated with $L$ is selfadjoint if it is semibounded, and if there exists a constant $K>0$ so that for all multi-indices $\alpha$ and $\beta$ with $|\alpha| \leqslant 2$,

$$
\left|a_{\alpha}^{(\beta)}(x)\right| \leqslant K^{|\beta|+1}(1+e|x|)^{|\alpha|-|\beta|},
$$

where $e=0$ or 1 .

REMARKS. 1. The condition (2.1) is, of course, a very restrictive condition on the coefficients. Elementary examples of ordinary differential operators which satisfy the conditions of the theorem are

$$
\left(x^{2} u^{\prime}\right)^{\prime}+i\left[x u^{\prime}+(x u)^{\prime}\right]
$$

and

$$
\left(\sin ^{2} x u^{\prime}\right)^{\prime}+i\left[\sin ^{2} x \cos x u^{\prime}+\left(\sin ^{2} x \cos x u\right)^{\prime}\right] .
$$

Elementary examples of partial differential operators are

$$
\partial_{1}|x|^{2} \partial_{1} u+\partial_{2} x_{2}^{2} \partial_{2} u
$$

and

$$
\partial_{1} \sin ^{2}\left(x_{1}+x_{2}\right) \partial_{1} u+\partial_{2} \cos ^{2} x_{2} \partial_{2} u
$$

Actually, it is not necessary to put any smoothness requirements on $a_{0}$, since once we have proved the essential selfadjointness under the conditions we have given, the essential selfadjointness will not change by the addition of a bounded measurable function.

2. As our proof will show, we could have also obtained results for first order operators by making use of Nussbaum's result in [2]. However, we have refrained from commenting about this since Paul R. Chernoff [1] has obtained much better results for first order systems by making more sophisticated use than we have been able to do of the circle of ideas connected with analytic vectors. His techniques also work for certain second order semibounded elliptic operators.

The estimates necessary to prove the above theorem are based in part upon the following

Lemma. There exists a constant $K>0$ so that for every nonnegative integer $s$ and every multi-index $\alpha$, 


$$
\int_{R^{n}}\left|(1+|x|)^{s} \partial^{\alpha} \exp \left(-|x|^{2}\right)\right|^{2} d x \leqslant[K(|\alpha|+s)]^{|\alpha|+s} \text {. }
$$

3. Proof of the theorem. For $a \in R^{n}$, let $u_{a}=\exp \left(-|x+a|^{2}\right)$ and set $u=\left(c_{1} u_{a_{1}}, \ldots, c_{n} u_{a_{n}}\right)$, where $c_{k}$ is a complex number. We claim that $u$ is in the domain of $L_{0}^{j}$ for every positive integer $j$. The proof is more or less standard. Let $\phi$ be a real valued function in $C_{0}^{\infty}\left(R^{n}\right)$ such that $0 \leqslant \phi \leqslant 1, \phi$ $=1$ for $|x| \leqslant 1$, and $\phi=0$ for $|x| \geqslant 2$. If we set $\phi_{k}(x)=\phi(x / k)$ it is clear that $L u \in L_{m}^{2}\left(R^{n}\right)$ and that $u_{k}=\phi_{k} u \in D\left(L_{0}\right)$. Further we have

$$
L u-L_{0} u_{k}=\left(1-\phi_{k}\right) L u+M_{k}^{1} u,
$$

where $M_{k}^{1}$ is a linear function in the derivatives of $\phi_{k}$ with $m \times m$ matrix coefficients which are at most of polynomial growth. Thus $M_{k}^{1} u \rightarrow 0$ in $L_{m}^{2}\left(R^{n}\right)$ and since clearly $\left(1-\phi_{k}\right) L u \rightarrow 0$ in this same space, we see that $u \in L_{0}$ and $L_{0} u=L u$.

Make the inductive hypothesis that $u \in L_{0}^{j}$ and that $v_{j} \equiv L_{0}^{j} u=N_{j} u$, where $N_{j}$ is an $m \times m$ matrix valued function so that it and each of its derivatives is at most of polynomial growth. Thus $L v_{j} \in L_{m}^{2}\left(R^{n}\right)$, and as before we have

$$
L v_{j}-L_{0} \phi_{k} v_{j}=\left(1-\phi_{k}\right) L v_{j}+M_{k}^{j} u,
$$

where $M_{k}^{j}$ has the same properties as $M_{k}^{1}$. Thus $u$ is in the domain of $L_{0}^{j+1}$ and $L_{0}^{j+1} u=L^{j+1} u$. This also shows that $L_{0}^{j+1} u=N_{j+1} u$, where $N_{j+1}$ has the same properties as $N_{j}$.

Let us make the inductive hypothesis that the differential operator $L^{j}$ may be written as

$$
L^{j}=\sum_{|\alpha| \leqslant 2 j} a_{j, \alpha} \partial^{\alpha}
$$

where $a_{j, \alpha} \in C^{\infty}\left(R^{n}\right)$, and for $1 \leqslant k \leqslant j$ and $2(k-1) \leqslant|\alpha| \leqslant 2 k$,

$$
\left|a_{j, \alpha}^{(\beta)}(x)\right| \leqslant \frac{4^{(j-1)(n+2)} K^{3 j-2+|\beta|}[(j-1) !]^{2}}{[(k-2) !]^{2}(k-1)^{|\alpha|-2(k-1)}} j^{|\beta|}(1+e|x|)^{|\alpha|-|\beta|},
$$

where $K$ is some suitable constant and, as in the hypothesis of the theorem, $e=0$ or 1 . We also make the convention that for $k=1$, the denominator on the right-hand side of (3.2) shall be 1 . Note that if $j=1$ then $a_{1, \alpha} \equiv a_{\alpha}$ and (3.1) and (3.2) are satisfied by hypothesis.

Using (3.1) we may write

$$
\begin{aligned}
L^{j+1} & =\sum_{|\alpha| \leqslant 2} a_{\alpha} \partial^{\alpha} \sum_{|\beta| \leqslant 2 j} a_{j, \beta} \partial^{\beta} \\
& =\sum_{|\beta| \leqslant 2 j} \sum_{|\alpha| \leqslant 2} \sum_{\nu \leqslant \alpha}\left(\begin{array}{l}
\alpha \\
\nu
\end{array}\right) a_{\alpha} a_{j, \beta}^{(\nu)} \partial^{\alpha+\beta-\nu} .
\end{aligned}
$$

For $|\alpha| \leqslant 2,|\beta| \leqslant 2 j$, and $\nu \leqslant \alpha$, let us set for fixed $\gamma,|\gamma| \leqslant 2(j+1)$, 


$$
a_{j+1, \gamma} \equiv \sum_{\alpha+\beta-\nu=\gamma}\left(\begin{array}{l}
\alpha \\
\nu
\end{array}\right) a_{\alpha} a_{j, \beta}^{(\nu)} .
$$

From Leibnitz' formula

$$
\left(a_{\alpha} a_{j, \beta}^{(\nu)}\right)^{(\mu)}=\sum_{\delta \leqslant \mu}\left(\begin{array}{l}
\mu \\
\delta
\end{array}\right) a_{\alpha}^{(\delta)} a_{j, \beta}^{(\nu+\mu-\delta)} .
$$

In (3.4) let us suppose, at first, that $2(k-1)<|\gamma| \leqslant 2 k, 1 \leqslant k \leqslant j$. Since $\alpha-\nu \geqslant 0$ and $|\alpha| \leqslant 2$, we must have $|\gamma|-2 \leqslant|\beta| \leqslant|\gamma|$. Thus the maximum value that can be taken on by $|\nu|$ is $|\beta|-|\gamma|+2$. If $|\beta| \geqslant 2(k-1)$ we have from (3.5) and the inductive hypothesis,

$$
\begin{aligned}
\left|\left(a_{\alpha} a_{j, \beta}^{(\nu)}\right)^{(\mu)}\right| \leqslant & \frac{4^{(j-1)(n+2)} K^{3(j+1)-2+|\mu|}[j !]^{2}}{[(k-2) !]^{2}(k-1)^{|\beta|-2(k-1)} j|\gamma|-|\beta|} \\
& \cdot(j+1)^{|\mu|}(1+e|x|)^{|\gamma|-|\mu|} \\
\leqslant & \frac{4^{(j-1)(n+2)} K^{3(j+1)-2+|\mu|}[j !]^{2}}{[(k-2) !]^{2}(k-1)^{|\gamma|-2(k-1)}} \\
& \cdot(j+1)^{|\mu|}(1+e|x|)^{|\gamma|-|\mu|} .
\end{aligned}
$$

If $|\beta|=2 k-3$, then the only possibility is $\nu=0$ and in this case we have

$$
\begin{aligned}
\left|\left(a_{\alpha} a_{j, \beta}^{(\nu)}\right)^{(\mu)}\right| & \leqslant \frac{4^{(j-1)(n+2)} K^{3(j+2)-2+|\mu|}[j !]^{2}}{[(k-3) !]^{2}(k-2) j^{2}} \cdot(j+1)^{|\mu|}(1+e|x|)^{|\gamma|-|\mu|} \\
& <\frac{4^{(j-1)(n+2)} K^{3(j+1)-2+|\mu|}[j !]^{2}}{[(k-2) !]^{2}(k-1)} \cdot(j+1)^{|\mu|}(1+e|x|)^{|\gamma|-|\mu|} .
\end{aligned}
$$

Since when $|\beta|=2 k-3$ we must have $|\gamma|=2 k-1$ (and $|\alpha|=2$ ) it follows that inequality (3.6) is satisfied in this case as well.

For given $\alpha, \nu$, and $\gamma, \beta$ is determined by the equation $\alpha+\beta-\nu=\gamma$. Hence, for $\gamma$ fixed we have the crude estimate

$$
\sum_{\alpha+\beta-\nu=\gamma}\left(\begin{array}{l}
\alpha \\
\nu
\end{array}\right) \leqslant \sum_{|\alpha| \leqslant 2} \sum_{\nu \leqslant \alpha}\left(\begin{array}{l}
\alpha \\
\nu
\end{array}\right) \leqslant 2^{2}(4)^{n} \leqslant 4^{n+2} .
$$

If we use this estimate in conjunction with (3.4) and (3.6) we see we have (3.2) with $j$ replaced by $(j+1)$.

It is still necessary to consider the case when $k=j+1$ so that $2 j<|\gamma|$ $\leqslant 2(j+1)$. Now, the maximum value for $|\beta|$ in (3.4) is $2 j$, and because of the present bound on $|\gamma|$, the minimum value for $|\beta|$ is $2 j-1$. As before, the maximum value which can be taken on by $|\nu|$ is $|\beta|-|\gamma|+2$. If one uses the inductive hypothesis and makes the same computations which lead to (3.6) one will get this inequality with $k$ replaced by $j+1$. It will then follow as before that (3.2) is valid with $j$ replaced by $j+1$, and $1 \leqslant k \leqslant j+1$. Further, it is clear that 


$$
L^{j+1}=\sum_{|\alpha| \leqslant 2(j+1)} a_{j+1, \alpha} \partial^{\alpha} .
$$

Thus the induction is complete.

We are now in a position to get estimates on the numbers $\left\|L_{0}^{j} u\right\|$. From (3.1) and (3.2) we see that there is a constant $K$ so that

$$
\begin{aligned}
\left\|L_{0}^{j} u\right\| \leqslant & K^{j}[(j-1) !]^{2} \\
& \cdot \sum_{k=1}^{j} \sum_{2(k-1) \leqslant|\alpha| \leqslant 2 k} \frac{\left\|(1+|x|)^{|\alpha|} \partial^{\alpha} u\right\|}{[(k-2) !]^{2}(k-1)^{|\alpha|-2(k-1)}} .
\end{aligned}
$$

Using $K$ as a generic constant we note first that $1+|x| \leqslant K\left(1+\left|x+a_{i}\right|\right), 1$ $\leqslant i \leqslant k$. Hence using the lemma we get for $|\alpha| \leqslant 2 k$,

$$
\begin{aligned}
\left\|(1+|x|)^{|\alpha|} \partial^{\alpha} u\right\| & \leqslant K \sum_{i=1}^{n}\left\|\left(1+\left|x+a_{i}\right|\right)^{|\alpha|} \partial^{\alpha} u_{a_{i}}\right\| \\
& \leqslant K(K k)^{2 k},
\end{aligned}
$$

and thus for $2(k-1) \leqslant|\alpha| \leqslant 2 k \leqslant 2 j$,

$$
\frac{\left\|(1+|x|)^{|\alpha|} \partial^{\alpha} u\right\|}{[(k-2) !]^{2}(k-1)^{|\alpha|-2(k-1)}} \leqslant K^{j}\left(\frac{k}{k-2}\right)^{2 k},
$$

where we have used Stirling's approximation in the last inequality. Making use of this estimate in (3.7) and using Stirling's approximation once more we see that there is a suitable constant $K$ so that $\left\|L_{0}^{j} u\right\| \leqslant K^{j} j^{2 j}$. Thus we have

$$
\sum_{j=1}^{\infty}\left\|L_{0}^{j} u\right\|^{-1 / 2 j}=\infty
$$

Since the Fourier transform of $\exp \left(-|x|^{2}\right)$ does not vanish, the linear manifold determined by its translates is dense in $L^{2}\left(R^{n}\right)$. Thus the linear manifold determined by the set of $u$ for which (3.8) is true is dense in $L_{m}^{2}\left(R^{n}\right)$. That is to say, $L_{0}$ has a total set of quasi-analytic vectors in the sense of Nussbaum. Hence we conclude from Nussbaum's theorem that $L_{0}$ is selfadjoint.

4. Proof of the lemma. Let us begin by supposing that $x$ is a real variable and $\beta$ is a nonnegative integer. By a well-known formula we have

$$
\begin{aligned}
\int_{-\infty}^{\infty}\left|x^{\beta} \exp \left(-x^{2}\right)\right|^{2} d x & =2 \int_{0}^{\infty} x^{2 \beta} \exp \left(-2 x^{2}\right) d x \\
& =\sqrt{\frac{\pi}{2}} \frac{1 \cdot 3 \cdot 5 \cdots(2 \beta-1)}{2^{2 \beta}}
\end{aligned}
$$

If $k$ is a nonnegative integer we recall that

$$
(d / d x)^{k} \exp \left(-x^{2}\right)=H_{k}(x) \exp \left(-x^{2}\right),
$$

where $H_{k}$ is the Hermite polynomial of order $k$. We also recall the recurrence 
formula

$$
H_{k+1}=2 x H_{k}-2 k H_{k-1}, \quad H_{-1}=0 .
$$

Let us make the inductive hypothesis that for every nonnegative integer $\beta$ and for every nonnegative integer $\alpha \leqslant k$,

$$
\begin{aligned}
& \int_{-\infty}^{\infty}\left|x^{\beta}\left(\frac{d}{d x}\right)^{\alpha} \exp \left(-x^{2}\right)\right|^{2} d x \\
& \leqslant(\alpha+1)^{2} \sqrt{\frac{\pi}{2}} \frac{1 \cdot 3 \cdot 5 \cdots(2(\alpha+\beta)-1)}{2^{2 \beta}}
\end{aligned}
$$

Formula (4.1) shows that (4.4) is true when $\alpha=0$. An elementary computation combined with (4.1) shows that (4.4) is also true when $\alpha=1$. Making use of (4.2), (4.3), the Schwarz inequality, and the inductive hypothesis (4.4) we get

$$
\begin{aligned}
& \int_{-\infty}^{\infty} \mid x^{\beta}\left(\frac{d}{d x}\right)^{k+1}\left.\exp \left(-x^{2}\right)\right|^{2} d x \\
& \leqslant 2^{2}\left\{\left[\int_{-\infty}^{\infty}\left|x^{\beta+1}\left(\frac{d}{d x}\right)^{k} \exp \left(-x^{2}\right)\right|^{2} d x\right]^{1 / 2}\right. \\
&\left.+k\left[\int_{-\infty}^{\infty}\left|x^{\beta}\left(\frac{d}{d x}\right)^{k-1} \exp \left(-x^{2}\right)\right|^{2} d x\right]^{1 / 2}\right\}^{2} \\
& \leqslant 2^{2} \sqrt{\pi / 2}\left\{(k+1)\left[1 \cdot 3 \cdots(2(k+\beta+1)-1) / 2^{2(\beta+1)}\right]^{1 / 2}\right. \\
&\left.+k^{2}\left[1 \cdot 3 \cdots(2(k+\beta-1)-1) / 2^{2 \beta}\right]^{1 / 2}\right\}^{2} .
\end{aligned}
$$

For $\beta \geqslant 1$ and $k \geqslant 1$ we have

$$
\begin{aligned}
\frac{1 \cdot 3 \cdots(2(k+\beta-1)-1)}{2^{2 \beta}} & =\frac{1 \cdot 3 \cdots(2(k+\beta+1)-1)}{2^{2 \beta}(2(k+\beta)-2) \cdots(2(k+\beta)+1)} \\
& \leqslant \frac{1}{(2 k)^{4}} \frac{1 \cdot 3 \cdots(2(k+\beta+1)-1)}{2^{2 \beta}} .
\end{aligned}
$$

Thus we have

$$
\begin{aligned}
\int_{-\infty}^{\infty}\left|x^{\beta}\left(\frac{d}{d x}\right)^{k+1} \exp \left(-x^{2}\right)\right|^{2} d x \\
\leqslant\left(k+1+\frac{1}{2}\right)^{2} \sqrt{\frac{\pi}{2}} \frac{1 \cdot 3 \cdots(2(k+\beta+1)-1)}{2^{2 \beta}} .
\end{aligned}
$$

For $\beta=0$ we use Parseval's equality

$$
\int_{-\infty}^{\infty}\left|\left(\frac{d}{d x}\right)^{k+1} \exp \left(-x^{2}\right)\right|^{2} d x=\int_{-\infty}^{\infty}\left|\left[\left(\frac{d}{d x}\right)^{k+1} \exp \left(-x^{2}\right)\right]^{\wedge}(\xi)\right|^{2} d \xi
$$


where

$$
\begin{aligned}
{\left[\left(\frac{d}{d x}\right)^{k+1} \exp \left(-x^{2}\right)\right]^{\wedge}(\xi) } & =\frac{1}{\sqrt{2 \pi}} \int_{-\infty}^{\infty} e^{-i \xi x}\left(\frac{d}{d x}\right)^{k+1} \exp \left(-x^{2}\right) d x \\
& =(i \xi)^{k+1} \exp \left(-\xi^{2} / 4\right) / \sqrt{ } 2
\end{aligned}
$$

This gives

$$
\begin{aligned}
\int_{-\infty}^{\infty}\left|\left[\left(\frac{d}{d x}\right)^{k+1} \exp \left(-x^{2}\right)\right]^{\wedge}(\xi)\right|^{2} d \xi & =\int_{0}^{\infty} \xi^{2(k+1)} \exp \left(-\xi^{2} / 2\right) d \xi \\
& =\sqrt{\pi / 2} 1 \cdot 3 \cdots(2(k+1)-1)
\end{aligned}
$$

so that (4.4) is true for every $\alpha$ and $\beta$.

Let us now complete the proof of the lemma; i.e., we estimate the integral

$$
\int_{R^{n}}\left|(1+|x|)^{s} \partial^{\alpha} \exp \left(-|x|^{2}\right)\right|^{2} d x
$$

We first notice that

$$
(1+|x|)^{2 s} \leqslant 2^{2 s}\left(1+|x|^{2 s}\right)
$$

and

$$
|x|^{2 s}=\sum_{|\beta|=s} c_{\beta} x^{2 \beta}, \quad \sum_{|\beta|=s} c_{\beta}=n^{s} .
$$

Hence we must estimate

$$
\text { (4.5) } \int_{R^{n}}\left|x^{\beta} \partial^{\alpha} \exp \left(-|x|^{2}\right)\right|^{2} d x=\prod_{j=1}^{n} \int_{-\infty}^{\infty}\left|x_{j}^{\beta_{j}}\left(\frac{d}{d x_{j}}\right)^{\alpha_{j}} \exp \left(-x_{j}^{2}\right)\right|^{2} d x_{j} \text {. }
$$

We have just previously estimated the integrals on the right so that the product is estimated by

$$
4^{2|\alpha|}\left(\frac{\pi}{2}\right)^{n / 2} \prod_{j=1}^{n} \frac{1 \cdot 3 \cdots\left(2\left(\alpha_{j}+\beta_{j}\right)-1\right)}{2^{2\left(\alpha_{j}+\beta_{j}\right)}}
$$

Using Stirling's estimate we may write

$$
\begin{aligned}
\frac{1 \cdot 3 \cdots\left(2\left(\alpha_{j}+\beta_{j}\right)-1\right)}{2^{2\left(\alpha_{j}+\beta_{j}\right)}} & =\frac{\left[2\left(\alpha_{j}+\beta_{j}\right)\right] !\left(\alpha_{j}+\beta_{j}\right)}{2^{2\left(\alpha_{j}+\beta_{j}\right)}\left(\alpha_{j}+\beta_{j}\right) ! 2^{\alpha_{j}+\beta_{j}-1}} \\
& \leqslant 2 e^{-\left(\alpha_{j}+\beta_{j}\right)}\left(\alpha_{j}+\beta_{j}\right)^{\alpha_{j}+\beta_{j}}
\end{aligned}
$$

Thus it follows that

$$
4^{2|\alpha|} e^{-(|\alpha|+s)}(2 \pi)^{n / 2}(|\alpha|+s)^{|\alpha|+s}
$$

is an estimate for the left side of (4.5). As a consequence we get the estimate 


$$
\left.\int_{R^{n}}|(1+|x|)|^{s} \partial^{\alpha} \exp \left(-|x|^{2}\right)\right|^{2} d x \leqslant(2 \pi)^{n / 2}\left(4^{2} n / e\right)^{|\alpha|+s}(|\alpha|+s)^{|\alpha|+s},
$$

which completes the proof.

\section{REFERENCES}

1. Paul R. Chernoff, Essential self-adjointness of powers of generators of hyperbolic equations, J. Functional Analysis 12 (1973), 401-414.

2. A. E. Nussbaum, Quasi-analytic vectors, Ark. Mat. 6 (1965), 179-191. MR 33 \#3105.

3. - A note on quasi-analytic vectors, Studia Math. 33 (1969), 305-309. MR 40 \#4781.

Department of Mathematics, Northwestern University, Evanston, Illinois 60201 